\title{
Factores que afectan a la estructura de los recubrimientos de cinc obtenidos por inmersión ${ }^{(\bullet)}$
}

\author{
P.R. Seré ${ }^{(*)}$, J.D. Culcasi ${ }^{(* *)}$, C.I. Elsner ${ }^{(*)}$ y A.R. Di Sarli ${ }^{(*)}$ \\ Resumen En el proceso de galvanizado por inmersión, la solidificación del recubrimiento constituye una etapa \\ compleja en la que, además de formarse compuestos intermetálicos de Al-Fe, Al-Fe-Zn y Fe-Zn, \\ tiene lugar la segregación de elementos aleantes insolubles como el antimonio. Ambos fenómenos \\ son, al menos parcialmente, indeseables ya que la fragilidad de los intermetálicos de Fe- $\mathrm{Zn}$ afecta a \\ la ductilidad del recubrimiento y la difusión del antimonio hacia la superficie causa su ennegreci- \\ miento. Con un simulador diseñado a escala de laboratorio se galvanizaron chapas de diferente rugo- \\ sidad sujetas a condiciones operativas también diferentes, analizándose el efecto de la rugosidad del \\ acero y de los parámetros del proceso sobre las características del recubrimiento. Los resultados \\ obtenidos demostraron que la rugosidad afecta tanto al espesor del recubrimiento, tamaño de grano y \\ textura del cinc como a la formación de out-bursts, mientras que los parámetros del proceso lo hacen \\ sobre la morfología del intermetálico $\mathrm{Fe}_{2} \mathrm{Al}_{5}$ y la segregación de antimonio.
}

Palabras clave: Acero galvanizado. Galvanizado por inmersión. Microestructura. Capa de aleación. Rugosidad. Recubrimientos de cinc.

\section{Factors affecting the hot-dip zinc coatings structure}

\begin{abstract}
Coating solidification during hot-dip galvanizing is a very complex process due to $\mathrm{Al}-\mathrm{Fe}, \mathrm{Al}-\mathrm{Fe}-\mathrm{Zn}$ and $\mathrm{Fe}-\mathrm{Zn}$ intermetallic compounds development. Fe- $\mathrm{Zn}$ intermetallics are brittle and detrimental for the coating ductility, while the diffusion towards the surface of a segregated insoluble alloying such as antimonium causes the sheet darkness. Steel sheets of different roughness were hot-dip galvanized under different operation conditions using a laboratory scale simulator. The effect of steel roughness and process parameters upon coating characteristics were analysed. Experimental results showed that the steel roughness affects the coating thickness, zinc grain size and texture as well as the out-bursts development, while the process parameters affects the $\mathrm{Fe}_{2} \mathrm{Al}_{5}$ morphology and antimonium segregation.
\end{abstract}

Keywords: Galvanized steel sheet. Hot-dip galvanizing. Microstructure. Alloy layer. Roughness. Zinc coatings.

\section{INTRODUCCIÓN}

Durante el proceso de galvanizado por inmersión, las reacciones químicas que tienen lugar entre el acero y el cinc líquido dan origen a la formación de diferentes fases. Así, sobre el sustrato crecen cuatro fases: $\Gamma(18-31 \% \mathrm{Fe}), \Gamma_{1}(19-24 \% \mathrm{Fe}), \delta$

(•) Trabajo recibido el día 31 de marzo de 1997.

(*) Centro de Investigación y Desarrollo en Tecnología de Pinturas (CIDEPINT). CIC-CONICET, Avda. 52, 121 y 122. CP 1900 - La Plata (Argentina).

(**) Dpto. de Mecánica. Facultad de Ingeniería. Universidad Nacional de La Plata. Calle 48 y 116. CP 1900. La Plata (Argentina).
$(8-13 \% \mathrm{Fe})$ y $\zeta(6-7 \% \mathrm{Fe})$. Por último, se forma una solución sólida de hierro en cinc (fase $\eta$ ) que disuelve alrededor del 0,04\% de hierro (1). Las mencionadas fases intermetálicas son duras y frágiles, razón por la cual el producto obtenido no es apto para el conformado, ya que enevitablemente se produciría la fisuración y desprendimiento del recubrimiento. La adición al baño de $0,1-0,3 \% \mathrm{Al}$ conduce a la rápida formación de una fase $\mathrm{Al}-\mathrm{Fe}$ que inhibe el crecimiento de los compuestos intermetálicos de Fe-Zn y, por lo tanto, mejora las propiedades mecánicas de la chapa galvanizada (2). Esta fase intermetálica ha sido identificada por algunos autores como $\mathrm{Fe}_{2} \mathrm{Al}_{5}$, mientras que otros sugieren que se trata de un compuesto ternario de $\mathrm{Fe}-\mathrm{Al}-\mathrm{Zn}$ 
(3). El carácter transitorio de esta película de inhibición tiene como consecuencia indeseable la nucleación y crecimiento localizado de compuestos intermetálicos de Fe-Zn (out-bursts), que emergen del acero base al romperse la capa de inhibición (4 y 5). El que se produzca o no este fenómeno depende de la temperatura y de la composición química del baño (fundamentalmente del contenido de aluminio), del tiempo de inmersión, de la velocidad de enfriamiento y de la condición superficial del sustrato (3 y 6), la cual tiene, además, efecto sobre las características de la película de cinc. La orientación cristalográfica (textura) de los granos de cinc depende, principalmente, de factores externos, tales como velocidad de enfriamiento, gradiente térmico y condición superficial del acero (7). Este parámetro ejerce a su vez una marcada influencia sobre la resistencia a la corrosión, la pintabilidad y la conformabilidad de la chapa $(8,9$ y 10$)$.

En el presente trabajo se evaluó el efecto de la rugosidad del sustrato, de la temperatura del baño y de la velocidad de enfriamiento sobre la morfología de la capa de inhibición, la tendencia a la formación de out-bursts y la orientación cristalográfica de los granos de cinc. Asimismo, se investigó el efecto de la rugosidad del acero base sobre el tamaño de los cristales y sobre el espesor de la película de cinc.

\section{PARTE EXPERIMENTAL}

Se galvanizaron flejes de acero, de $200 \times 60 \times 1$ $\mathrm{mm}$, cuya composición química se muestra en la tabla I. La rugosidad superficial de las muestras se modificó tratándolas con distintos abrasivos. Las condiciones operativas utilizadas fueron las siguientes: composición del baño (ver Tabla II); temperatura 470 ó $520^{\circ} \mathrm{C}$; tiempo de inmersión 30 $\mathrm{s}$; velocidad de enfriamiento entre 5 y $22{ }^{\circ} \mathrm{C} \mathrm{s}^{-1}$ (Tabla III).

TABLA I.- Composición química del acero base, en $\mathrm{p} / \mathrm{p} \%$

TABLE I.- Chemical composition of the base steel, $p / p \%$

\begin{tabular}{|cccccc|}
\hline $\mathrm{C}$ & $\mathrm{Mn}$ & $\mathrm{P}$ & $\mathrm{S}$ & $\mathrm{Si}$ & $\mathrm{Al}$ \\
\hline 0,060 & 0,250 & 0,015 & 0,015 & 0,020 & 0,040 \\
\hline
\end{tabular}

TABLA II.- Composición química del baño, en p/p \%

TABLE II.- Chemical composition of the zinc bath, $p / p \%$

\begin{tabular}{|cccc|}
\hline $\mathrm{Sb}$ & $\mathrm{Al}$ & $\mathrm{Fe}$ & $\mathrm{Zn}$ \\
\hline 0,09 & 0,20 & 0,04 & 99,67 \\
\hline
\end{tabular}

TABLA III.- Condiciones experimentales operativas

TABLE III.- Experimental operational conditions

\begin{tabular}{|cccc|}
\hline Muestra & $\begin{array}{c}\text { Temperatura, } \\
{ }^{\circ} \mathrm{C}\end{array}$ & $\begin{array}{c}\text { Rugosidad } \\
R_{\mathrm{a}}, \mu \mathrm{m}\end{array}$ & $\begin{array}{c}\text { Velocidad de } \\
\text { enfriamiento, } \\
{ }^{\circ} \mathrm{C} \mathrm{s}^{-1}\end{array}$ \\
\hline A & 470 & 0,95 & 5,0 \\
B & 470 & 1,30 & 6,6 \\
C & 470 & 2,30 & 8,4 \\
D & 470 & 0,95 & 22,0 \\
E & 520 & 0,95 & 6,3 \\
F & 520 & 1,30 & 7,0 \\
G & 520 & 2,30 & 11,0 \\
\hline
\end{tabular}

La morfología y composición de las distintas fases se caracterizaron mediante microscopía electrónica de barrido (SEM), analizador EDAX y difracción de rayos $X$ (XRS). Se realizaron observaciones metalográficas según dos planos: transversal y paralelo a la superficie de la chapa. Las muestras para observación transversal se atacaron con Nital $1 \%$ + ácido crómico, mientras que, en las observaciones paralelas a la superficie, la película de cinc fue disuelta en solución de ácido sulfúrico + inhibidor para evitar la disolución del intermetálico Fe-Al. Mediante XRS se determinó la intensidad de las reflexiones correspondientes a los planos cristalográficos de cinc y se las comparó con los valores dados por la norma ASTM 4-831, correspondiente a una muestra de polvos con orientación al azar. Para cada plano analizado se calculó la siguiente relación:

$$
R_{\text {(hk.l) }}=\left(I_{(\text {hk.l) }} / \Sigma I_{(\text {hiki.li) }}\right) \times 100
$$

donde $I_{(\text {hk.l) }}$ es la intensidad de la reflexión y $\Sigma I_{\text {(hiki.li) }}$ la suma de las intensidades de todas las reflexiones analizadas. La misma relación se realizó a partir de los datos obtenidos de la norma ASTM 4-831 (muestra patrón $R_{\mathrm{p}(\mathrm{hk} . \mathrm{l})}$ ).

Por último, se calculó el coeficiente de textura, $C T_{\text {(hk.l) }}$, definido como:

$$
C T_{(\mathrm{hk} .1)}=R_{(\mathrm{hk} . \mathrm{l})} / R_{\mathrm{p}(\mathrm{hk} . \mathrm{l})}
$$

Un $C T_{(\mathrm{hk} .1)}>1$ indica una orientación preferencial del plano correspondiente (hk.l) con respecto a la exhibida por la muestra patrón.

\section{RESULTADOS Y DISCUSIÓN}

\subsection{Topografía del sustrato}

Para una temperatura del baño de $470{ }^{\circ} \mathrm{C}$, la disminución del tamaño de los cristales de cinc con el 
aumento de la rugosidad del acero mostrada en la figura 1a) se atribuye a que al aumentar la superficie específica también lo hace el número de sitios de nucleación, factor este último que conduce a la obtención de cristales de cinc más pequeños. Por otro lado, al ser mayor la cinética de la reacción entre el hierro y el cinc a $520^{\circ} \mathrm{C}$ que en el caso anterior, se presume la existencia no sólo de una disolución más rápida en las zonas con mayor energía superficial (por ej., los picos superficiales del hierro) sino también que esto conduciría a una disminución de la superficie específica y, por lo tanto, del número de centros de nucleación y crecimiento. Tal presunción ha permitido explicar el motivo por el cual, a esta temperatura, el tamaño de grano del cinc depositado era similar al de las muestras de menor rugosidad ensayadas a $470{ }^{\circ} \mathrm{C}$; sin embargo, se continúa investigando con el objeto de encontrar mayor evidencia experimental que la apoye. Otro aspecto a tener en cuenta es que al incrementarse la altura y densidad de picos (es decir, la rugosidad) también aumenta el arrastre de cinc líquido y, en consecuencia, el espesor de la película de cinc (Fig. 1b).

En cuanto a la influencia de la rugosidad del acero sobre la textura del recubrimiento, las muestras con mayor rugosidad presentaron un mayor coeficiente de textura para los planos (00.2) (Fig. 2 ), indicando que un aumento de la superficie específica promueve el desarrollo de planos basales paralelos al sustrato.

En concordancia con lo publicado por Shah y col. (11), de la comparación de los resultados experimentales descritos para los parámetros anteriores (espesor, textura y tamaño de grano) puede inferirse que la relación existente entre los mismos es inversa entre el desarrollo de los planos basales y el tamaño de grano, y directa entre aquél y el espesor del recubrimiento.

Con referencia a la formación de la capa de inhibición, identificada mediante XRS como $\mathrm{Fe}_{2} \mathrm{Al}_{5}$, no se detectaron diferencias de la morfología y tamaño de sus cristales al cambiar la rugosidad (Fig. 3). Según Gutmman y col. (4), esta fase intermetálica está compuesta por dos subcapas; una de ellas, constituida por cristales equiáxicos de aproximadamente $60 \mathrm{~nm}$ de diámetro, compacta y muy delgada en contacto con el sustrato, mientras que la segunda, depositada sobre aquélla, es más gruesa y está formada por cristales aplastados de 300 a $600 \mathrm{~nm}$ de diámetro y aproximadamente $200 \mathrm{~nm}$ de espesor. La cinética del proceso de nucleación y crecimiento de la capa delgada es muy rápida, del orden de una fracción de segundo, en tanto que la de la superior, correspondiente a la segunda etapa de crecimiento, es mucho más lenta debido a que está controlada por la difusión del hierro a través de la ya solidificada capa adherida al acero. Por otro lado, mientras el mencionado proceso para la capa delgada está

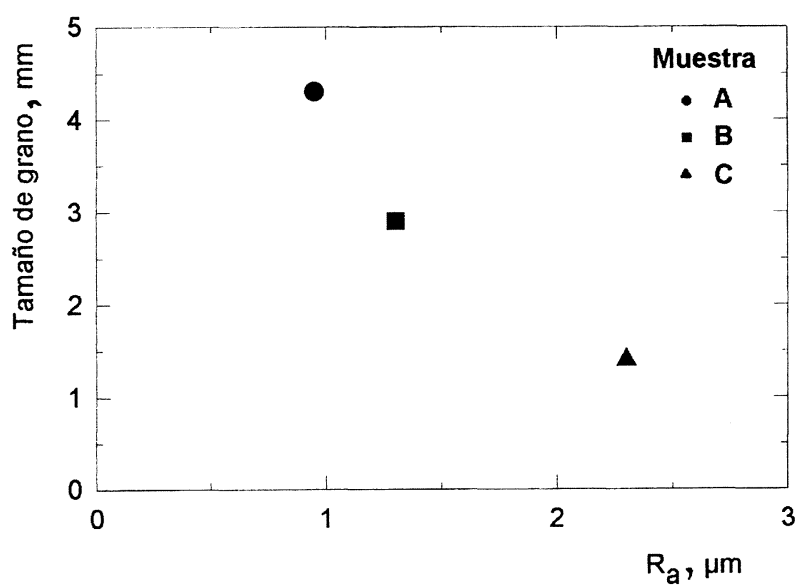

a

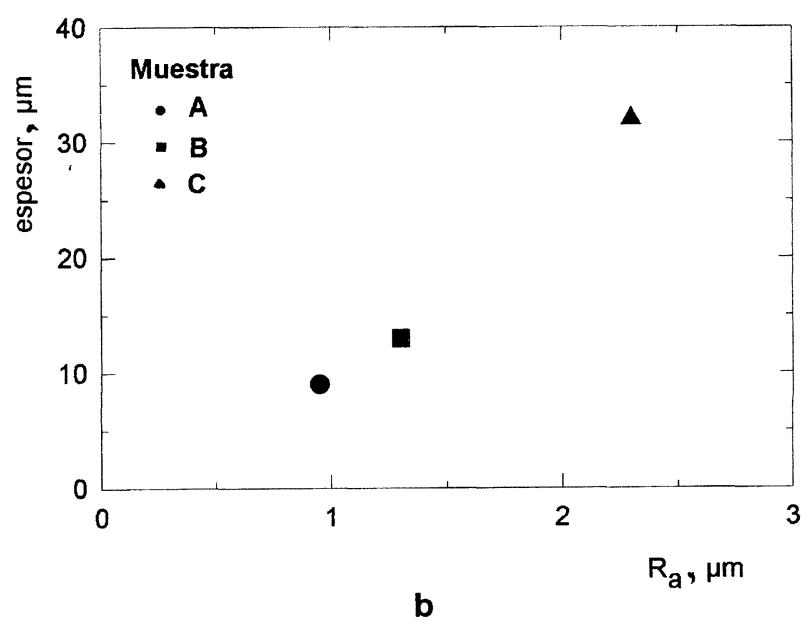

FIG. 1.- a) Tamaño de los cristales, y b) espesor del recubrimiento de cinc frente a la rugosidad media del acero $\left(R_{\mathrm{a}}\right)$.

FIG. 1.- a) Crystals size, and b) thickness of the zinc coating vs. steel average roughness $\left(\mathrm{R}_{\mathrm{a}}\right)$.

controlado por las características estructurales del acero, en la superior exhibe una morfología y orientación cristalográfica al azar. Este hecho explicaría la falta de correlación entre la rugosidad del sustrato y la morfología del intermetálico de Fe-Al. En lo que respecta a la formación de out-bursts, originada por la rotura localizada de la capa de inhibición, se encontró que el aumento de rugosidad estaría acompañado por una tendencia al crecimiento localizado de fases de Fe-Zn (Fig. 4).

\subsection{Temperatura del baño}

Si bien en los ensayos realizados a ambas temperaturas, la morfología del intermetálico 


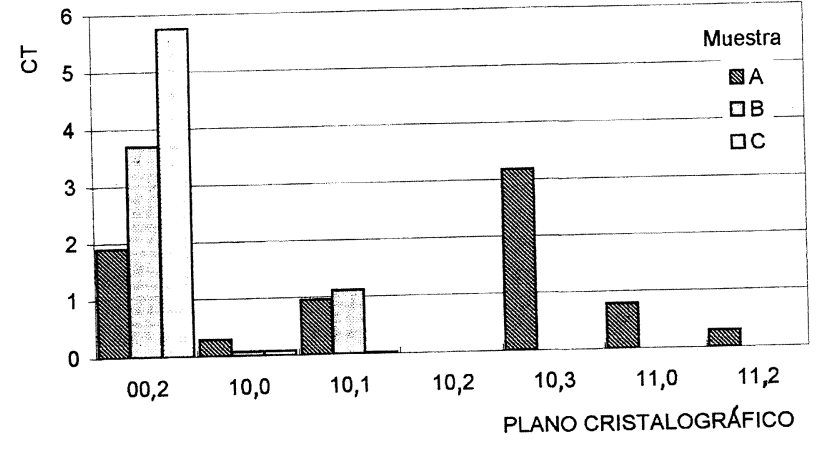

FIG. 2.- Coeficiente de textura (CT) frente a $R_{\mathrm{a}}$ para las muestras: A $\left(R_{\mathrm{a}}=0,95 \mu \mathrm{m}\right), \mathrm{B}\left(R_{\mathrm{a}}=1,30\right.$ $\mu \mathrm{m})$ у C $\left(R_{\mathrm{a}}=2,30 \mu \mathrm{m}\right)$.

FIG. 2.- Texture coefficient (CT) vs. $\mathrm{R}_{\mathrm{a}}$ for samples: $A\left(\mathrm{R}_{\mathrm{a}}=0.95 \mu \mathrm{m}\right), B\left(\mathrm{R}_{\mathrm{a}}=1.30 \mu \mathrm{m}\right)$ and $C\left(\mathrm{R}_{\mathrm{a}}=2.30 \mu \mathrm{m}\right)$.
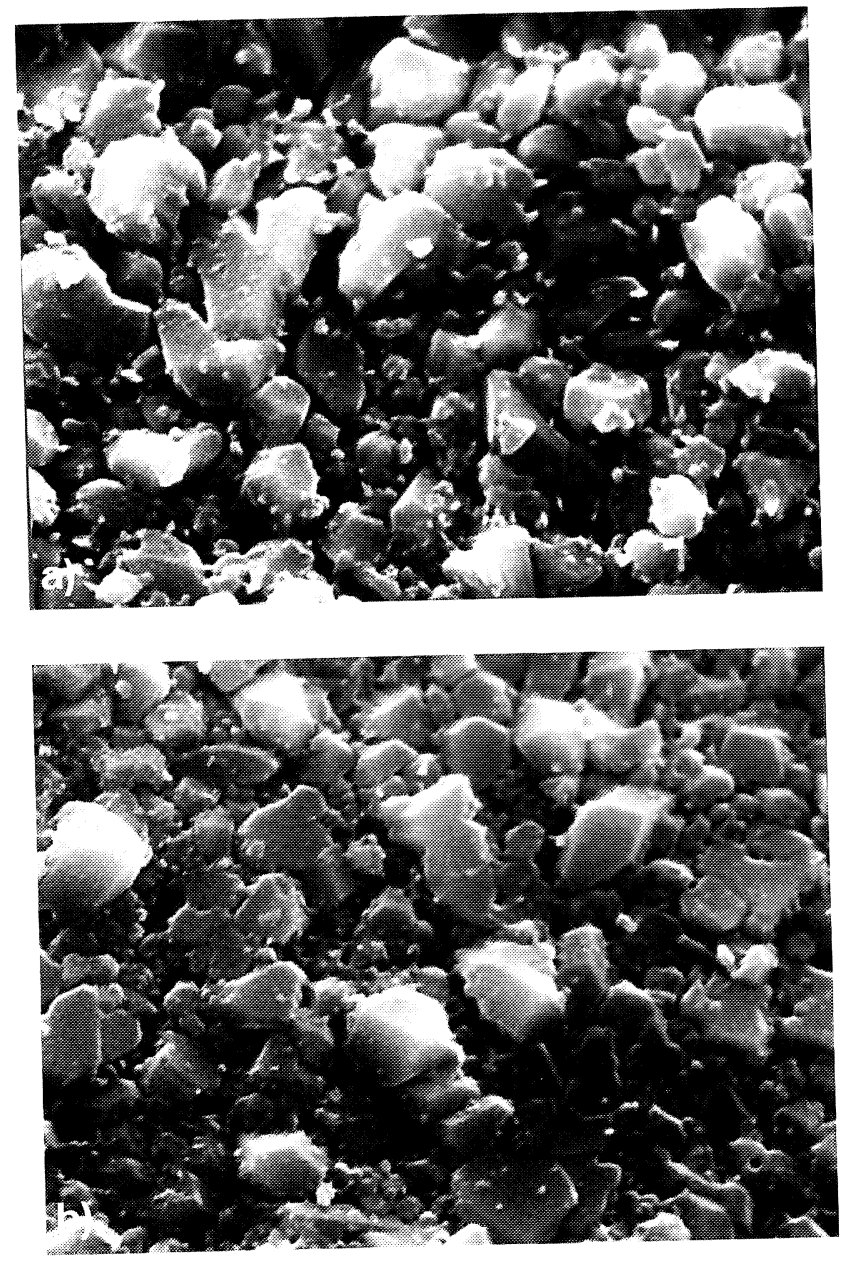

FIG. 3.- Morfología de la capa de inhibición frente a $R_{\mathrm{a}}$ para las muestras: a) $\mathrm{E}\left(R_{\mathrm{a}}=0,95 \mu \mathrm{m}\right.$. $\times 7.700)$, y b) G $\left(R_{\mathrm{a}}=2,30 \mu \mathrm{m} . \times 7.700\right)$.

(Reproducida en imprenta al $80 \%$ )

FIG. 3.- Inhibition layer morphology vs. $\mathrm{R}_{\mathrm{a}}$ for samples: a) $E\left(\mathrm{R}_{\mathrm{a}}=0.95 \mu \mathrm{m} . \times 7,700\right)$, and $\left.b\right)$ $G\left(\mathrm{R}_{\mathrm{a}}=2.30 \mu m . \times 7,700\right)$.

(Printing reduction $80 \%$ )
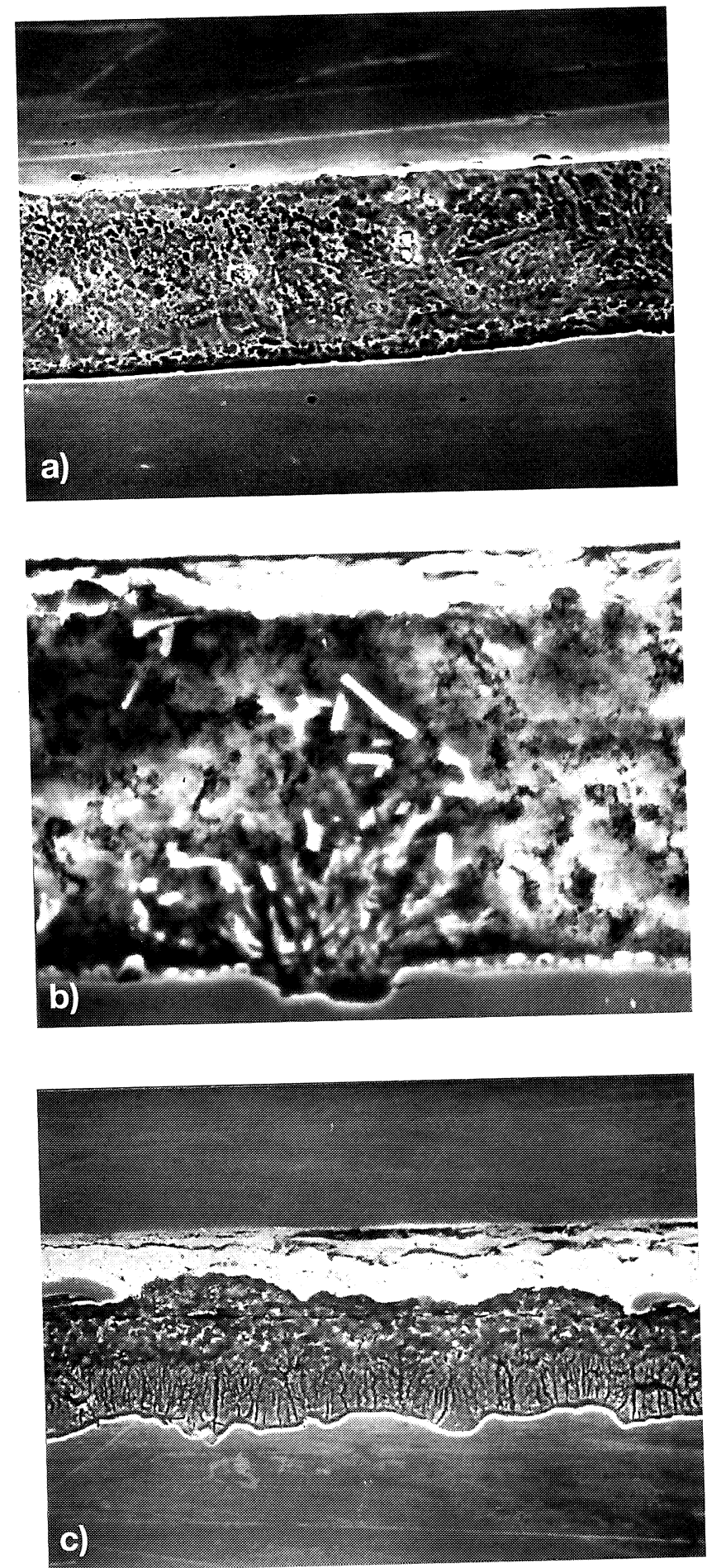

FIG. 4.- Desarrollo de out-bursts frente a $R_{\mathrm{a}}$ para las muestras: a) A $\left(R_{\mathrm{a}}=0,95 \mu \mathrm{m} . \times 500\right)$, b) B $\left(R_{\mathrm{a}}\right.$ $=1,30 \mu \mathrm{m} . \times 1.490)$ y c) C $\left(R_{\mathrm{a}}=2,30 \mu \mathrm{m} . \times 500\right)$. (Reproducida en imprenta al $80 \%$ )

FIG. 4.-Out-bursts development vs. $\mathrm{R}_{\mathrm{a}}$ for samples: a) $\left.A\left(\mathrm{R}_{\mathrm{a}}=0.95 \mu m . \times 500\right), b\right) B\left(\mathrm{R}_{\mathrm{a}}=\right.$ $1.30 \mu \mathrm{m} . \times 1,490)$ and c) $C\left(\mathrm{R}_{\mathrm{a}}=2.30 \mu \mathrm{m} . \times 500\right)$. (Printing reduction $80 \%$ )

$\mathrm{Fe}_{2} \mathrm{Al}_{5}$ fue granular, resultó evidente que, a mayor temperatura, se producía un mayor crecimiento de 
esta fase, alcanzándose gran desarrollo de los cristales que se distribuyen homogéneamente en forma y tamaño sobre la superficie (Fig. 5a). A menor temperatura se observó no sólo la presencia de cristales más pequeños sino también de sitios donde el intermetálico crece preferencialmente, hecho a partir del cual se infirió que, en su primera etapa, el crecimiento no es homogéneo sino que se desarrolla heterogéneamente a lo largo de la superficie (Fig. 5 b). Las muestras galvanizadas a $520{ }^{\circ} \mathrm{C}$ mostraron un crecimiento de fases de $\mathrm{Fe}-\mathrm{Zn}$, mientras que a $470{ }^{\circ} \mathrm{C}$ no se detectó la presencia de outbursts.

En cuanto a la orientación cristalográfica del recubrimiento, no se encontró ninguna dependencia entre la temperatura del baño y la textura.
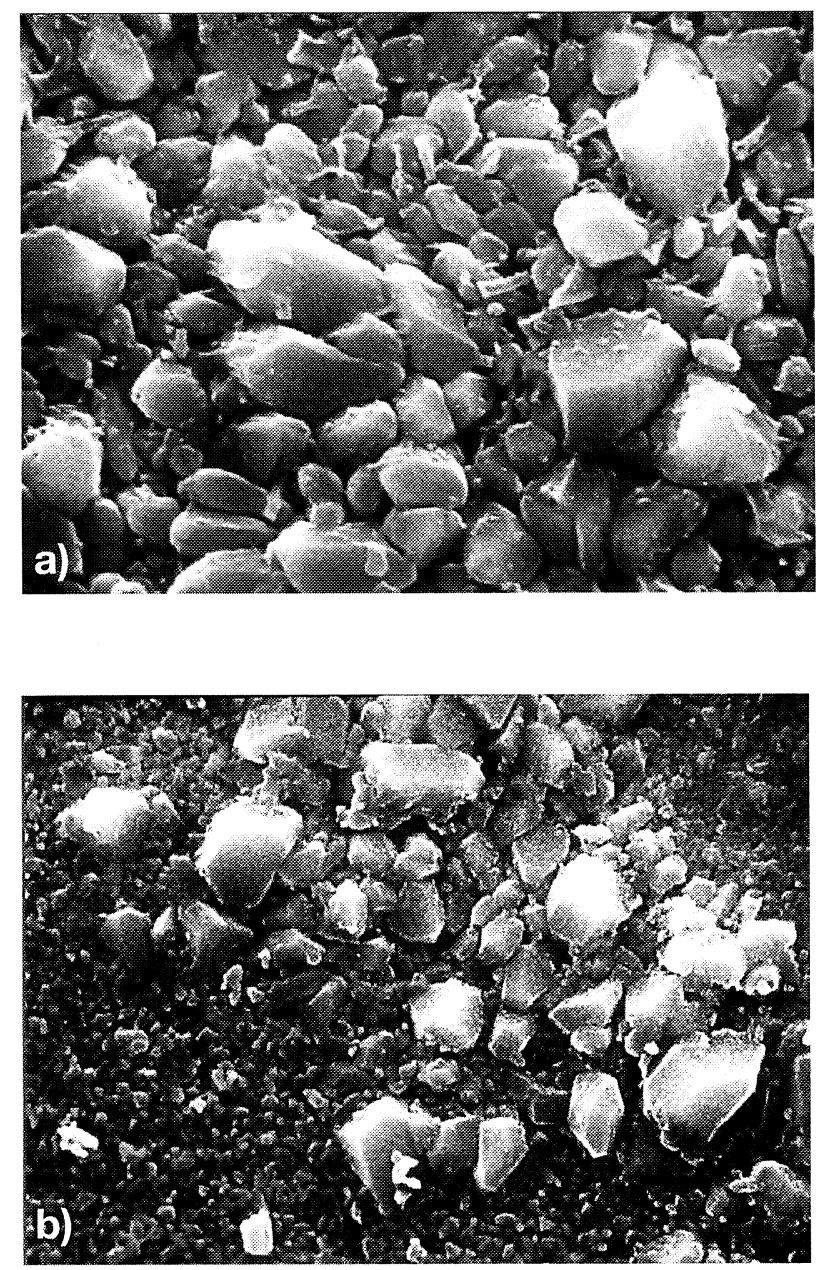

FIG. 5.- Morfología de la capa de inhibición en función de la temperatura del baño de cinc $(T)$ para las muestras: a) $\mathrm{E}\left(T=520{ }^{\circ} \mathrm{C} . \times 7.700\right)$, y b) A $\left(T=470{ }^{\circ} \mathrm{C} . \times 7.700\right)$.

(Reproducida en imprenta al $80 \%$ )

FIG. 5.- Inhibition layer morphology vs. zinc bath temperature (T) for samples: a) $E\left(\mathrm{~T}=520{ }^{\circ} \mathrm{C}\right.$. $\times 7,700)$, and $b) A\left(\mathrm{~T}=470^{\circ} \mathrm{C} . \times 7,700\right)$.

(Printing reduction $80 \%$ )

\subsection{Velocidad de enfriamiento}

La velocidad de enfriamiento tiene una marcada influencia sobre la formación del intermetálico $\mathrm{Fe}_{2} \mathrm{Al}_{5}$. Así, al aumentar ésta de 5 a $22{ }^{\circ} \mathrm{C} \mathrm{s}^{-1}$, el tamaño de los cristales de la capa de inhibición disminuye (Fig. 6a); además, los estudios realizados con EDAX en las muestras enfriadas a mayor velocidad detectaron, sobre el intermetálico, un agregado acicular formado mayormente por antimonio (Fig. 6b). Este hecho es muy importante ya que el antimonio segregado a la superficie es el responsable del ennegrecimiento de la chapa en cortos tiempos de exposición a la intemperie, perjuicio que podría evitarse si el antimonio segregara sobre el intermetálico y no sobre la superficie. Los estudios del efecto sobre la textura determinaron que con el
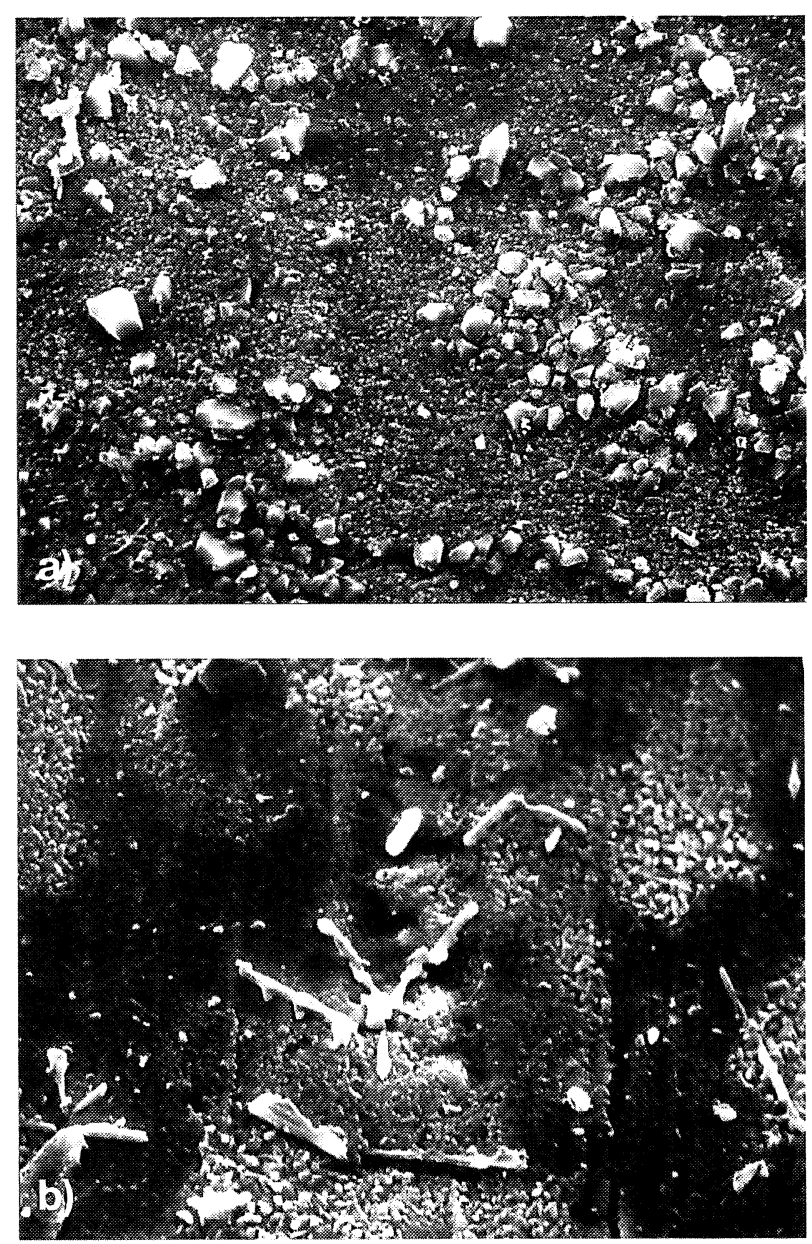

FIG. 6.- Morfología de la capa de inhibición frente a la velocidad de enfriamiento $\left(v_{\mathrm{e}}\right)$ para las muestras: a) $\mathrm{A}\left(v_{\mathrm{e}}=5{ }^{\circ} \mathrm{C} \mathrm{seg}^{-1} \cdot \times 2.500\right)$, y b) $\mathrm{D}\left(v_{\mathrm{e}}=\right.$ $\left.22^{\circ} \mathrm{C} \mathrm{seg}^{-1} \cdot \times 2.500\right)$.

(Reproducida en imprenta al $80 \%$ )

FIG. 6.- Inhibition layer morphology vs. cooling rate $\left(\mathrm{v}_{\mathrm{e}}\right)$ for samples: a) $A\left(\mathrm{v}_{\mathrm{e}}=5{ }^{\circ} \mathrm{C} \mathrm{seg}^{-1}\right)$, and b) $D\left(\mathrm{v}_{\mathrm{e}}=22{ }^{\circ} \mathrm{C} \mathrm{seg}^{-1}\right)$.

(Printing reduction $80 \%$ ) 
incremento de la velocidad de enfriamiento aumenta el coeficiente de textura de los planos basales (Fig. 7).

\section{CONCLUSIONES}

La rugosidad del acero es un parámetro que influye significativamente sobre las características del recubrimiento. En tal sentido, al aumentar dicha rugosidad se incrementa el espesor del recubrimiento, disminuye el tamaño de grano de cinc y se desarrolla una textura basal más definida. Existe, además, una mayor tendencia a la formación de out-bursts.

Elevadas temperaturas del baño producen un excesivo crecimiento de los cristales de la capa de inhibición y gran tendencia a la formación de outbursts. No se encontró dependencia entre la temperatura del baño y la textura de los cristales de cinc.

El empleo de un sistema eficiente de extracción del calor de las muestras a la salida del baño (por ej., spray de agua) produce un aumento de la velocidad de enfriamiento y, como consecuencia de ello, no sólo una disminución del tamaño de los

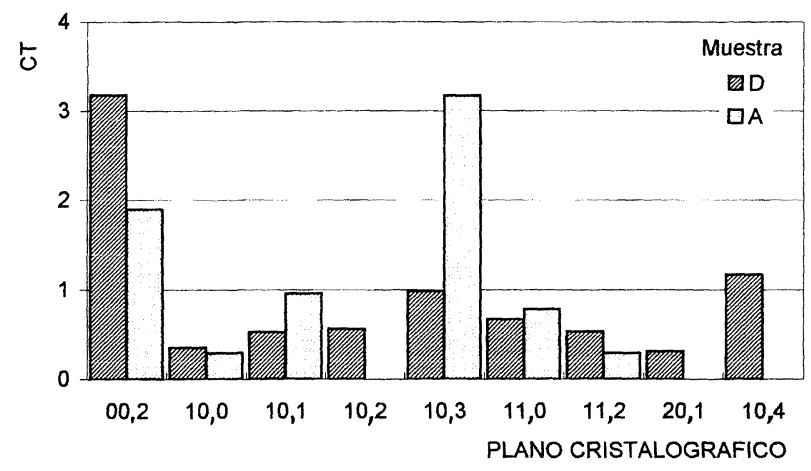

FIG. 7.- Coeficiente de textura (CT) frente a $v_{\mathrm{e}}$ para las muestras: $\mathrm{D}\left(v_{\mathrm{e}}=22{ }^{\circ} \mathrm{C} \mathrm{seg}^{-1}\right)$ y $\mathrm{A}\left(v_{\mathrm{e}}=\right.$ $5{ }^{\circ} \mathrm{C}$ seg $\left.^{-1}\right)$.

FIG. 7.- Texture coefficient $v s . \mathrm{v}_{\mathrm{e}}$ for samples: $D\left(\mathrm{v}_{\mathrm{e}}=22{ }^{\circ} \mathrm{C} \mathrm{seg}^{-1}\right)$ and $\mathrm{A}\left(\mathrm{v}_{\mathrm{e}}=5^{\circ} \mathrm{C} \mathrm{seg}^{-1}\right)$. granos del intermetálico $\mathrm{Fe}_{2} \mathrm{Al}_{5}$ sino que también promueve la orientación cristalográfica de los planos basales paralelos al sustrato. Además, al aumentar la segregación de antimonio sobre el intermetálico disminuye el ennegrecimiento de la superficie de la chapa.

\section{Agradecimientos}

Los autores del trabajo agradecen a la Comisión de Investigaciones Científicas de la Provincia de Buenos Aires (CIC), al Consejo Nacional de Investigaciones Científicas y Técnicas (CONICET) y a la Universidad Nacional de La Plata (UNLP) el apoyo económico brindado para su ejecución.

\section{REFERENCIAS}

(1) Cook, D.C. y Grant, R.G. Galvatech '95 Conf. Proc. Chicago (EE.UU.), 1995: 497-508.

(2) ASM Handbook. Ninth ed. 13, Hot-Dip Coatings. 1992. 432.

(3) Lin, C.S. y MeshiI, M. Galvatech '95 Conf. Proc. Chicago (EE.UU), 1995: 335-342.

(4) Gutmman, M., Lepretre, Y., Aubry, A., Roch, M.J., Moreau, T., Drillet, P., Mataigne, J.M. y Baudin, H. Galvatech '95 Conf. Proc. Chicago (EE.UU.), 1995: 295307.

(5) Hisamatsu, Y. Galvatech '89 Conf. Proc. Tokyo (Japón), 1989: 3-12.

(6) MascheK, W., Hayes, S.P. y Marder, A.R. Galvatech '95 Conf. Proc. Chicago (EE.UU.), 1995: 309-318.

(7) Chang, S. y Shin, J.C. Corros. Sci., 36 (8), 1994: 1.4251.436.

(8) Millan, J.L. J. Mater. Eng. Performance, 1 (2), 1992: 275-283.

(9) Lindsay, J.H., Paluch, R.F., Nine, H.D., Miller, V.R. y O’KeEFe, T.J. Plating Surf. Finish., Mar., 1989: 62-69.

(10) Shaffer, S.J., NoJima, W.E., Skalpelos, P.N. y Morris, J.W. TMS Symposium on Zinc-Based Steel Coating Systems Conf. Proc. Detroit (EE.UU.), 1990: 251-261.

(11) SHAH, S.R.H., Dilewijns, J.A. y JONES, R.D. Galvatech ' 92 Conf. Proc., Amsterdam (Países Bajos), 1992: 105111. 